\title{
Myocardium injury biomarkers predict prognosis of critically ill coronavirus disease 2019 (COVID-19) patients
}

\author{
Liang Cao ${ }^{1 \#}$, Sha Zhang ${ }^{1,2 \#}, \mathrm{Xi} \mathrm{Luo}^{3 \#}$, Enxin Wang ${ }^{4}$, Yang Bai ${ }^{5}$, Zhe $\mathrm{Li}^{6}$, Feng $\mathrm{Li}^{1}$, Jing Ma ${ }^{1}$, Haitao Liu ${ }^{7}$ \\ ${ }^{1}$ Department of Traditional Chinese Medicine, Xijing Hospital, Fourth Military Medical University, Xi'an, China; ${ }^{2}$ Department of Basic Medicine, \\ Shaanxi University of Chinese Medicine, Xianyang, China; ${ }^{3}$ Xi'an People's Hospital (Xi'an Fourth Hospital), Xi'an, China; ${ }^{4}$ Department of Medical \\ Affairs, Air Force Hospital of Western Theater Command, Chengdu, China; ${ }^{5}$ Department of Neurosurgery, General Hospital of Northern Theater \\ Command (General Hospital of Shenyang Military Command), Shenyang, China; ${ }^{6}$ Department of First Clinical Medicine, Affiliated Hospital of \\ Shaanxi University of Chinese Medicine, Xianyang, China; ${ }^{7}$ Department of Cardiology, Xijing Hospital, Fourth Military Medical University, Xi'an, \\ China; Department of Infectious Diseases, Hubei Women \& Children Healthcare Hospital (Guanggu District), Wuhan, China \\ Contributions: (I) Conception and design: L Cao, S Zhang, X Luo, F Li, J Ma, H Liu; (II) Administrative support: Z Li, F Li, J Ma, H Liu; \\ (III) Provision of study materials or patients: H Liu; (IV) Collection and assembly of data: L Cao, S Zhang, X Luo, Z Li; (V) Data analysis and \\ interpretation: L Cao, S Zhang, X Luo, E Wang, F Li, J Ma, H Liu; (VI) Manuscript writing: All authors; (VII) Final approval of manuscript: All \\ authors. \\ \#These authors contributed equally to this work. \\ Correspondence to: Haitao Liu. Department of Cardiology, Xijing Hospital, Fourth Military Medical University, Xi'an, China. \\ Email: cardio@fmmu.edu.cn; Jing Ma. Department of Traditional Chinese Medicine, Xijing Hospital, Fourth Military Medical University, Xi'an, \\ China. Email: jingma@fmmu.edu.cn; Feng Li. Department of Traditional Chinese Medicine, Xijing Hospital, Fourth Military Medical University, \\ Xi'an, China. Email: fenglitcm@163.com.
}

Background: The coronavirus disease 2019 (COVID-19) pandemic is a once-in-century crisis to public health. Although the pathogen for COVID-19, the severe acute respiratory syndrome coronavirus-2 (SARS$\mathrm{CoV}-2$ ), has been identified, the pandemic is still ongoing. The critically ill COVID-19 patients account for most disease-associated death; thus, there is an urgent need to identify prognostic factors that would help determine therapeutic approaches.

Methods: In this study, we retrospectively analyzed the clinical and laboratory findings in 100 critically ill COVID-19 patients in Hubei Women \& Children Healthcare Hospital (Guanggu District), of whom 22 patients died in hospital, and 78 patients survived.

Results: We found that age, lymphocyte count, and total bilirubin concentration were an independent prognostic factor for critically ill COVID-19 patients. Of particular importance, we observed a significant elevation of myocardium injury biomarkers, including CK-MB, high-sensitivity cardiac troponini I (hs-c'TnI), and $\mathrm{Mb}$, in the non-survivor group. These myocardium injury biomarkers appeared to correlate with the time of survival, and two multivariate models have suggested hs-cTnI was a novel prognostic factor with a sensitivity of $75.0 \%$ and a specificity of $84.9 \%$.

Conclusions: Altogether, our study highlighted the prognostic significance of myocardium injury biomarkers in critically ill COVID-19 patients. Monitoring myocardium injury biomarkers would predict patient survival and guide therapeutic strategy.

Keywords: Coronavirus disease 2019 (COVID-19); severe acute respiratory syndrome coronavirus-2 (SARS$\mathrm{CoV}-2$ ); prognostic factor; myocardium injury biomarkers; high-sensitivity cardiac troponini I (hs-cTnI)

Submitted Oct 12, 2020. Accepted for publication Nov 17, 2020.

doi: 10.21037/apm-20-2112

View this article at: http://dx.doi.org/10.21037/apm-20-2112 


\section{Introduction}

The global outbreak of coronavirus disease 2019 (COVID-19) is a significant threat to human health. To date, more than 200 countries and more than 10 million people are affected by the disease. The pathogen for COVID-19 is severe acute respiratory syndrome coronavirus-2 (SARS-CoV-2), and it belongs to the $\beta$-type coronavirus family $(1,2)$. Genomic studies have shown that SARS-CoV-2 is a single-stranded RNA virus composed of $30 \mathrm{~kb}$ nucleotides. These genes encode four major structural proteins: spike protein $(\mathrm{S})$, membrane protein $(\mathrm{M})$, an envelope protein (E), and nucleocapsid protein $(\mathrm{N})$. The $\mathrm{S}$ protein is of particular interest among these proteins because it gives the virus a crownlike appearance and mediates the interaction with the angiotensin-converting enzyme 2 (ACE2) $(3,4)$. This procedure allows the entry of SARS-CoV-2 into the host cells, and ACE2 is defined as a putative receptor for SARS-CoV-2. To this end, the $\mathrm{S}$ protein-specific antibody is believed to elicit a protective effect against SARS-CoV-2 infection. The transfusion of convalescent plasma collected from recovered COVID-19 patients works through the neutralization of virus particles by the $S$ protein-specific antibody $(5,6)$.

Interestingly, the genomic homogeneity between SARS-CoV-2 and SARS-CoV underlies their overlapping biological properties $(7,8)$. The clinical manifestations and radiologic features of COVID-19 are like SARS, which appears in 2003. The patients manifest as fever, nonproductive cough, shortness of breath, and acute respiratory failure. However, increasing lines of evidence suggested that COVID-19 patients also presented with injuries in the brain, liver, kidney, and heart (9-12). Preliminary autopsy studies have shown that virus particles exist in these organs, which leads to inflammatory cell infiltration and tissue damage (13). In agreement with this, abnormalities in aspartate aminotransferase (AST), alanine aminotransferase (ALT), creatinine, and blood urea nitrogen (BUN) could be frequently observed in critically ill COVID-19 patients $(14,15)$. These changes tend to be associated with poor prognosis. Notably, cardiac injury associated with SARSCoV-2 infection is also presented in a group of critically ill COVID-19, and there are preliminary evidence showing potential risks of increased in-hospital death in COVID-19 patients presented with abnormalities in cardiac injury biomarkers (16). However, the number of COVID-19 patients in these studies is relatively small and the significance of myocardium injury during SARS-CoV-2 infection remains controversial.

This study retrospectively analyzed the clinical and laboratory features of 100 critically ill COVID-19 patients in Wuhan. We reported that serum biomarkers reflecting myocardium injury were significantly increased in most deceased cases. We propose that the myocardium damage caused by the virus attack may be a prognostic factor for COVID-19. We present the following article in accordance with the STARD reporting checklist (available at http:// dx.doi.org/10.21037/apm-20-2112).

\section{Methods}

\section{Patient enrollment}

In this retrospective cohort, study cases were obtained from a consecutive historical cohort of 115 patients diagnosed with critically ill COVID-19 in the Intensive Care Unit (ICU) of Hubei Women \& Children Healthcare Hospital (Guanggu Hospital District) between February 19th and April 17th in 2020. The critically ill COVID-19 was diagnosed in compliance with the New Coronavirus Pneumonia Diagnosis and Treatment Program (6th edition) published by the National Health Commission of China. Detailed criteria was listed as follows: (I) patients presenting as respiratory failure that requires mechanical ventilatrion; (II) patients presenting as shock; (III) patients presenting as multiple organs dysfunction that requires ICU admission. Patients' clinical data were reviewed using the Electronic Medical Records System and spreadsheets. Twelve patients with a malignant tumor and three dead patients during preclinical emergency treatment were excluded. Therefore, 100 eligible critically ill COVID-19 patients were included in this study. The study was conducted in accordance with the Declaration of Helsinki (as revised in 2013). The Ethics Medical Committee approved this study of Hubei Women \& Children Healthcare Hospital (No.2020-FYGG-011). Written consent was obtained from each participant.

\section{Data collection}

The diagnosis of COVID-19 follows the "New Coronavirus Pneumonia Diagnosis and Treatment Program (6th Edition)" issued by the National Health Commission of China (http://www.nhc.gov.cn/). All patients were laboratory-confirmed cases defined as a positive result for SARS-CoV-2 through real-time PCR assay of throat swab specimens. Critically ill patients were defined as those 
admitted to the ICU and required mechanical ventilation or had a fraction of inspired oxygen $\left(\mathrm{FiO}_{2}\right)$ of at least $60 \%$ or more. Identification of critically ill patients was achieved by reviewing and analyzing admission logs and histories from all available electronic medical records and patient care resources.

We extracted data on age, sex, chronic comorbidity (chronic cardiovascular disease, chronic pulmonary disease, cerebrovascular disease, diabetes), clinical symptoms, and laboratory findings on admission from electronic medical records. A chest CT examination was assessed, and two experienced independent radiologists reviewed each chest CT image. Radiologic abnormalities were determined with extensively documented characteristics, including groundglass opacities (GGOs) and consolidation. Laboratory assessments consisted of a complete blood count, blood chemical analysis, assessment of liver and renal function, hsCRP, and myocardium injury enzymes.

\section{Measurement of myocardium injury biomarkers}

The myocardium injury enzymes were measured by using a commercial available kit. Briefly, fresh blood samples were centrifugated at room temperature and the supernatant were carefully removed. $500 \mu \mathrm{l}$ of supernatant was mixed with equal volume of reaction buffer and reagent. After $15 \mathrm{~min}$ of incubation, the mixture was loaded on quantitative immunoassay analyzer to measure $\mathrm{Mb}, \mathrm{CK}-\mathrm{MB}$ and hscTnI concentrations, respectively.

\section{Treatments and follow-ups}

The critically ill COVID-19 patients were treated following China CDC recommendations. Briefly, all patients were empirically treated with oral arbidol (200 $\mathrm{mg}$, three times per day for no more than ten days) and Lianhua Qingwen capsule (two capsules, three times per day). For respiratory support, tracheal intubation and invasive mechanical ventilation were performed if the patient's oxygen saturation lower than $93 \%$ after receiving one to two hours of noninvasive ventilation. The lung-protective ventilation strategy that combined using low tidal volume $(4-6 \mathrm{~mL} / \mathrm{kg}$ ideal body weight) and higher levels of positive endexpiratory pressure $\left(\mathrm{PEEP},>10 \mathrm{cmH}_{2} \mathrm{O}\right)$ was used. The end-inspiratory plateau pressure was restricted to less than $30 \mathrm{cmH}_{2} \mathrm{O}$ to avoid ventilation-associated lung injury. The $\mathrm{FiO}_{2}$ was adjusted to the PEEP level to support oxygen saturation over $88 \%$. A lung recruitment maneuver was used when necessary.

After indicated treatment, patients met the following criteria considered to discharge from the hospital: body temperature returned to normal for more than three days; improved respiratory symptoms; a significant improvement in pulmonary imaging; two consecutive negative results of throat swab tests.

\section{Statistical analysis}

Categorical variables are described as the frequency and percentage, and continuous variables are described as the median with interquartile range (IQR). As the primary endpoint, patient survival was defined as the time from admission to the date of death or hospital discharge in one month. The univariate or multivariate Cox regression model was adopted to analyze the prognostic factors. Two multivariate regression models separately adjusted the prognostic ability of these myocardium injury biomarkers. Finally, the predictive values of different cardiac parameters were compared using receiver operating characteristic (ROC) curves. Statistical analyses were conducted using SPSS software version 22.0, and $\mathrm{P}$ values $<0.05$ (2-tailed) were considered significant.

\section{Results}

\section{Patient characteristics and laboratory findings}

For the 100 enrolled critically ill patients, the median age was 71 (IQR, 57-90) years. 45 (45\%) men and 55 (55\%) women in the study cohort. Twenty-two critically ill COVID-19 patients died during hospitalization, and the estimated mortality rate was $22 \%$. Relevant patient characteristics were summarized in Table 1 . The survivors tended to be younger, while there was no difference in the incidence of death between men and women. Although the absolute number of COVID-19 patients with chronic comorbidities in the survivor group was higher than that in the non-survivor group, the percentages of patients with hypertension, diabetes, coronary heart disease, and COPD were comparable within the two groups (Table 1).

For laboratory findings, there was a statistic difference in the white blood cell (WBC) count, lymphocyte count, and neutrophil count between the survivors and the nonsurvivors, in which the lower-level of lymphocyte was more 
Table 1 Baseline characteristics and laboratory findings of the participants

\begin{tabular}{|c|c|c|c|}
\hline Characteristics & Total $(n=100)$ & Non-survivor $(n=22)$ & Survivor $(n=78)$ \\
\hline Gender (men/women) & $45(45.0) / 55$ (55.0) & $12(54.5) / 10(45.5)$ & 33 (42.3)/45 (57.7) \\
\hline Hypertension & $60(60.0)$ & $11(50)$ & $49(62.8)$ \\
\hline Diabetes & $30(30.0)$ & $6(27.3)$ & $24(30.7)$ \\
\hline COPD & $6(6.0)$ & $2(9.1)$ & $4(5.1)$ \\
\hline WBC $\left(10^{9} / \mathrm{L}\right)^{\S}$ & $6.0(4.7-7.5)$ & $7.8(6.3-11.5)$ & $5.4(4.6-7.1)$ \\
\hline Lymphocyte $\left(10^{9} / L\right)^{\S}$ & $1.3(0.8-1.7)$ & $0.7(0.5-0.8)$ & $1.4(1.0-1.8)$ \\
\hline Neutrophil $\left(10^{9} / \mathrm{L}\right)^{\S}$ & $2.7(2.7-5.4)$ & $6.3(5.3-10.1)$ & $3.4(2.6-4.6)$ \\
\hline ALT (U/L) & $17.0(12.8-28.3)$ & $23.3(15.2-46.6)$ & $16.7(12.1-27.7)$ \\
\hline AST $(U / L)^{\S}$ & $19.3(14.6-29.3)$ & $34.1(23.3-54.4)$ & $18.1(14.0-26.6)$ \\
\hline Total bilirubin $(\mu \mathrm{mol} / L)^{\S}$ & $9.9(6.9-13.8)$ & $17.4(9.1-24.7)$ & $9.2(6.9-12.7)$ \\
\hline Albumin (g/L) & 36.0 (31.2-39.8) & $30.0(25.9-33.9)$ & $37.8(33.7-40.2)$ \\
\hline $\mathrm{BUN}(\mathrm{mmol} / \mathrm{L})^{\S}$ & $4.6(3.7-6.2)$ & $9.1(5.3-12.8)$ & $4.5(3.6-5.5)$ \\
\hline Creatinine $(\mu \mathrm{mol} / \mathrm{L})^{\S}$ & $67.0(55.9-80.4)$ & $73.2(53.3-123.9)$ & $66.7(55.9-75.5)$ \\
\hline $\mathrm{hsCRP}(\mathrm{mg} / \mathrm{L})^{\S}$ & $4.3(1.0-39.4)$ & $82.6(41.1-203.0)$ & $2.1(0.7-6.1)$ \\
\hline CK-MB $(\mu \mathrm{g} / \mathrm{L})^{\S}$ & $0.9(0.6-1.5)$ & $3.7(1.9-9.2)$ & $0.8(0.5-1.1)$ \\
\hline
\end{tabular}

Data are mean $\pm \mathrm{SD}$ or $\mathrm{n}(\%)$, or median (IQR). ${ }^{\S}$, variables with significant differences between non-survivors and survivors. COPD, chronic obstructive pulmonary diseases; WBC, white blood cell; ALT, alanine aminotransferase; AST, aspartate aminotransferase; BUN, blood urea nitrogen; hsCRP, high sensitive C reaction protein; CK-MB, creatine kinase isoenzyme-MB; hs-cTnl, high-sensitivity cardiac troponini I; $\mathrm{Mb}$, myoglobin; IQR, interquartile range.

frequently observed in the non-survivors. Biochemical analysis showed an approximately two-fold increase in serum AST (rather than ALT), total bilirubin, and BUN in the non-survivor group. Although the serum creatinine's median value between the two groups appeared comparable, statistical analysis showed the creatinine concentration in the non-survivor group was significantly higher than that in the survivor group. In agreement with earlier studies, the level of inflammatory indicators, including highly sensitive C-reactive protein (hsCRP), was markedly elevated.

Given ACE2 is expressed in the myocardium, it is reasonable to believe SARS-CoV-2 infection causes myocardium injury. Indeed, we notice biomarkers showing myocardium injury were ubiquitously increased in the non-survivors. These biomarkers included myoglobin $(\mathrm{Mb})$, creatine kinase isoenzyme-MB (CK-MB), and highsensitivity cardiac troponini I (hs-cTnI), in which hs-cTnI was the most specific indicator for myocardium injury. Of note, the altitude of hs-cTnI abnormality is the most evident (8.87-, 4.6-, and 5.86-fold for hs-cTnI, CK-MB, and $\mathrm{Mb}$, respectively).

\section{Prognostic factors for critically ill COVID-19 patients}

To find whether these statistics different findings were associated with prognosis, we performed a Cox regression 
Table 2 Univariate and multivariate Cox regression analyses for identifying prognostic factors

\begin{tabular}{|c|c|c|c|c|}
\hline Variables & \multicolumn{2}{|c|}{ Univariate analyses } & \multicolumn{2}{|c|}{ Multivariate analyses } \\
\hline Age, per year increase & $1.07(1.03-1.12)$ & 0.002 & $1.20(1.00-1.43)$ & 0.049 \\
\hline Gender male (ref to female) & $1.24(0.53-2.91)$ & 0.615 & - & - \\
\hline Hypertension & $0.69(0.29-1.64)$ & 0.400 & - & - \\
\hline Coronary heart disease & $1.24(0.41-3.73)$ & 0.701 & - & - \\
\hline COPD & $1.70(0.39-7.36)$ & 0.477 & - & - \\
\hline Lymphocyte, per $10^{9} / \mathrm{L}$ increase & $0.11(0.04-0.36)$ & $<0.001$ & $0.01(0.01-0.44)$ & 0.029 \\
\hline Neutrophil, per $10^{9} / \mathrm{L}$ increase & $1.18(1.08-1.29)$ & $<0.001$ & $0.76(0.55-1.04)$ & 0.086 \\
\hline ALT, per $1 \mathrm{U} / \mathrm{L}$ increase & $1.02(1.00-1.04)$ & 0.057 & - & - \\
\hline AST, per $1 \mathrm{U} / \mathrm{L}$ increase & $1.07(1.04-1.10)$ & $<0.001$ & $1.02(0.97-1.97)$ & 0.466 \\
\hline Total bilirubin, per $1 \mu \mathrm{mol} / \mathrm{L}$ increase & $1.10(1.05-1.15)$ & $<0.001$ & $1.21(1.02-1.44)$ & 0.029 \\
\hline Albumin, per $1 \mathrm{~g} / \mathrm{L}$ increase & $0.86(0.79-0.94)$ & 0.001 & $1.10(0.98-1.37)$ & 0.371 \\
\hline BUN, per $1 \mathrm{mmol} / \mathrm{L}$ increase & $1.27(1.10-1.46)$ & 0.001 & $0.74(0.31-1.80)$ & 0.513 \\
\hline Creatinine, per 1 umol/L increase & $1.03(1.01-1.04)$ & 0.008 & $1.05(0.98-1.13)$ & 0.197 \\
\hline hsCRP, per $1 \mathrm{mg} / \mathrm{L}$ & $1.01(1.00-1.01)$ & 0.001 & $1.01(1.00-1.01)$ & 0.148 \\
\hline
\end{tabular}

For univariate analysis of chronic comorbidities, patients without hypertension, diabetes, coronary heart disease, and COPD were set for references. HR, hazard ratio; COPD, chronic obstructive pulmonary diseases; ALT, alanine aminotransferase; AST, aspartate aminotransferase; BUN, blood urea nitrogen; hsCRP, high sensitive $\mathrm{C}$ reaction protein.

analysis. We used both univariate analyses and multivariate analyses to maximize the analysis accuracy and verify independent prognostic factors. Inconsistent with earlier reports, age was an independent, unfavorable prognostic factor for critically ill COVID-19 patients. However, we did not find a correlation between sex and chronic comorbidities with prognosis. As shown in Table 2, univariate Cox regression analyses showed that lymphocyte count, neutrophil count, AST, total bilirubin, albumin, BUN, creatinine, and hsCRP concentrations appeared to correlate to prognosis. To be specific, higher levels of lymphocyte count and albumin may predict a favorable prognosis of critically ill COVID-19 patients ( $\mathrm{HR}<1.0)$, and this has been confirmed in other literature. However, higher levels of the neutrophil count, AST, total bilirubin, BUN, creatinine, and hsCRP may predict unfavorable prognosis $(\mathrm{HR}>1.0)$.

Multivariate Cox regression analysis was done to verify whether these indexes were an independent prognostic factor of critically ill COVID-19. However, it led to conflicting results. We found the lymphocyte count was an independent prognostic factor because the multivariate analysis elicited an $\mathrm{HR}$ value $<1.0$ and $\mathrm{P}$ value $<0.05$. This showed that the odds of in-hospital death were higher in patients with lymphopenia. Meanwhile, the total bilirubin also functioned as a prognostic factor of critically ill COVID-19 patients. As shown in Table 2, multivariate analysis indicated an HR value of 1.21 and a $P$ value of 0.029 for total bilirubin, suggesting that the total bilirubin concentration negatively correlated with patients' prognosis. Although univariate analysis showed a possible correlation of neutrophil count, AST, albumin, BUN, creatinine, and hsCRP and patients' prognosis, the multivariable logistic regression model did not support that they served as independent prognostic factors. 
Table 3 Myocardium injury biomarkers predict the prognosis of critically ill COVID-19 patients

\begin{tabular}{lcc}
\hline \multirow{2}{*}{ Variables } & \multicolumn{2}{c}{ Cox regression analyses } \\
\cline { 2 - 3 } & $\mathrm{HR}(95 \% \mathrm{Cl})$ & P value \\
\hline Univariate analyses & $1.09(1.04-1.14)$ & $<0.001$ \\
CK-MB, per $1 \mathrm{\mu g} / \mathrm{L}$ increase & $1.00(1.00-1.01)$ & 0.004 \\
hs-cTnl, per $1 \mathrm{ng} / \mathrm{mL}$ increase & $1.00(1.00-1.01)$ & 0.002 \\
Mb, per $1 \mathrm{ng} / \mathrm{mL}$ increase & & \\
Multivariate Model 1 & $24.2(4.0-145.7)$ & $<0.001$ \\
CK-MB, per $1 \mathrm{\mu g} / \mathrm{L}$ increase & $9.48(1.20-74.9)$ & 0.033 \\
hs-cTnl, per $1 \mathrm{ng} / \mathrm{mL}$ increase & $1.00(1.00-1.01)$ & 0.657 \\
Mb, per $1 \mathrm{ng} / \mathrm{mL}$ increase & & \\
Multivariate Model 2 & $1.22(0.73-2.05)$ & 0.446 \\
CK-MB, per $1 \mathrm{\mu g} / \mathrm{L}$ increase & $1.00(1.00-1.01)$ & 0.045 \\
hs-cTnl, per $1 \mathrm{ng} / \mathrm{mL}$ increase & $0.75(0.56-0.99)$ & 0.049 \\
\hline Mb, per $1 \mathrm{ng} / \mathrm{mL}$ increase & &
\end{tabular}

COVID-19, coronavirus disease 2019; CK-MB, creatine kinase isoenzyme-MB; hs-cTnl, high-sensitivity cardiac troponini I; Mb, myoglobin.

\section{Identifying myocardium injury biomarkers as prognostic factors}

We were particularly interested in evaluating the significance of myocardium injury biomarkers in critically ill COVID-19 patients. Because Table 1 showed that Mb, CK-MB, and hs-cTnI were increased in the non-survivors, this has prompted us to evaluate whether these biomarkers predicted prognosis. Indeed, univariate Cox regression analysis showed that despite the HR value was of borderline significance, all the myocardium injury biomarkers tended to correlate with prognosis (Table 3).

Two multivariate analysis models were established to adjust myocardium injury biomarkers' prognostic value for patient survival (Table 3). In model 1, we included independent prognostic factor (age, lymphocyte count, total bilirubin), and myocardium injury biomarkers in the analysis. The result showed that the CK-MB (adjusted $\mathrm{HR}=24.2, \mathrm{P}<0.001$ ) and the hs-cTnI (adjusted HR =9.48, $\mathrm{P}=0.033)$ were independent prognostic factors, except for $\mathrm{Mb}$ (adjusted HR $=1.00, \mathrm{P}=0.657$ ). Moreover, in model 2 , age, lymphocyte count, total bilirubin, myocardium injury biomarkers, together with a neutrophil count, AST, albumin, BUN, creatinine, and hsCRP that correlate to prognosis were included. However, this multivariate analysis model revealed that the $\mathrm{Mb}$ (adjusted $\mathrm{HR}=0.75, \mathrm{P}=0.049$ ) and the hs-cTnI (adjusted HR $=1.00, \mathrm{P}=0.045$ ), but not $\mathrm{CK}-\mathrm{Mb}$ (adjusted $\mathrm{HR}=1.22, \mathrm{P}=0.446$ ), were independent prognostic factors.

\section{Myocardium injury biomarkers predict patient survival}

The fore-mentioned univariate and multivariate Cox regression analysis data highlighted that myocardium injury biomarkers were independent prognostic factors of critically ill COVID-19 patients; thus, it was reasonable to anticipate patients with myocardium injury would have an unfavorable prognosis. We retrospectively tracked the therapeutic outcomes of critically ill COVID-19 patients, as shown in Figure 1, the median time from admission to in-hospital death in critically ill patients with abnormal CK-MB was 7.0 (IQR 5.4-8.6) days, which was significantly less than those with normal CK-MB (Figure $1 A$, not reached, $\mathrm{P}<0.001$ ). However, in comparison with patients with normal hscTnI, those with a higher level of hs-cTnI exhibited a markedly shorter survival [26 (IQR 13.1-38.9) days vs. not reached, Figure $1 B, \mathrm{P}<0.001]$. This could be recaptured in the $\mathrm{Mb}$ subgroup, in which patients with a higher level of $\mathrm{Mb}$ associated with poor prognosis [15 (IQR 4.4-25.6) days vs. not reached, Figure $1 C, \mathrm{P}<0.001]$.

To determine these myocardium injury biomarkers' sensitivity and specificity in predicting prognosis, we analyzed the ROC curves. As we have expected, the area under ROC curves (AUC) in the hs-cTnI group was the most dominant [0.80 (95\% CI, 0.62-0.98)] because hscTnI was a relatively favorable biomarker of myocardium injury. As shown in Figure 2, the hs-cTnI reached a 75.0\% sensitivity, a specificity of $84.9 \%$. Compared to hs-cTnI, the AUC of $\mathrm{Mb}$ and CK-MB curves reached $0.72(95 \%$ CI, 0.50-0.95) and 0.68 (95\% CI, 0.45-0.92), respectively. These two myocardium injury biomarkers had similar specificity (94.5\% vs. $98.6 \%$ ), while $\mathrm{Mb}$ appeared to be more sensitive in predicting the prognosis than CK-MB (50.0\% vs. $37.5 \%)$.

\section{Discussion}

Although the lung is the most common target for COVID-19, critically ill patients may manifest multiple organ dysfunction, or even sepsis shock (17). Patients 

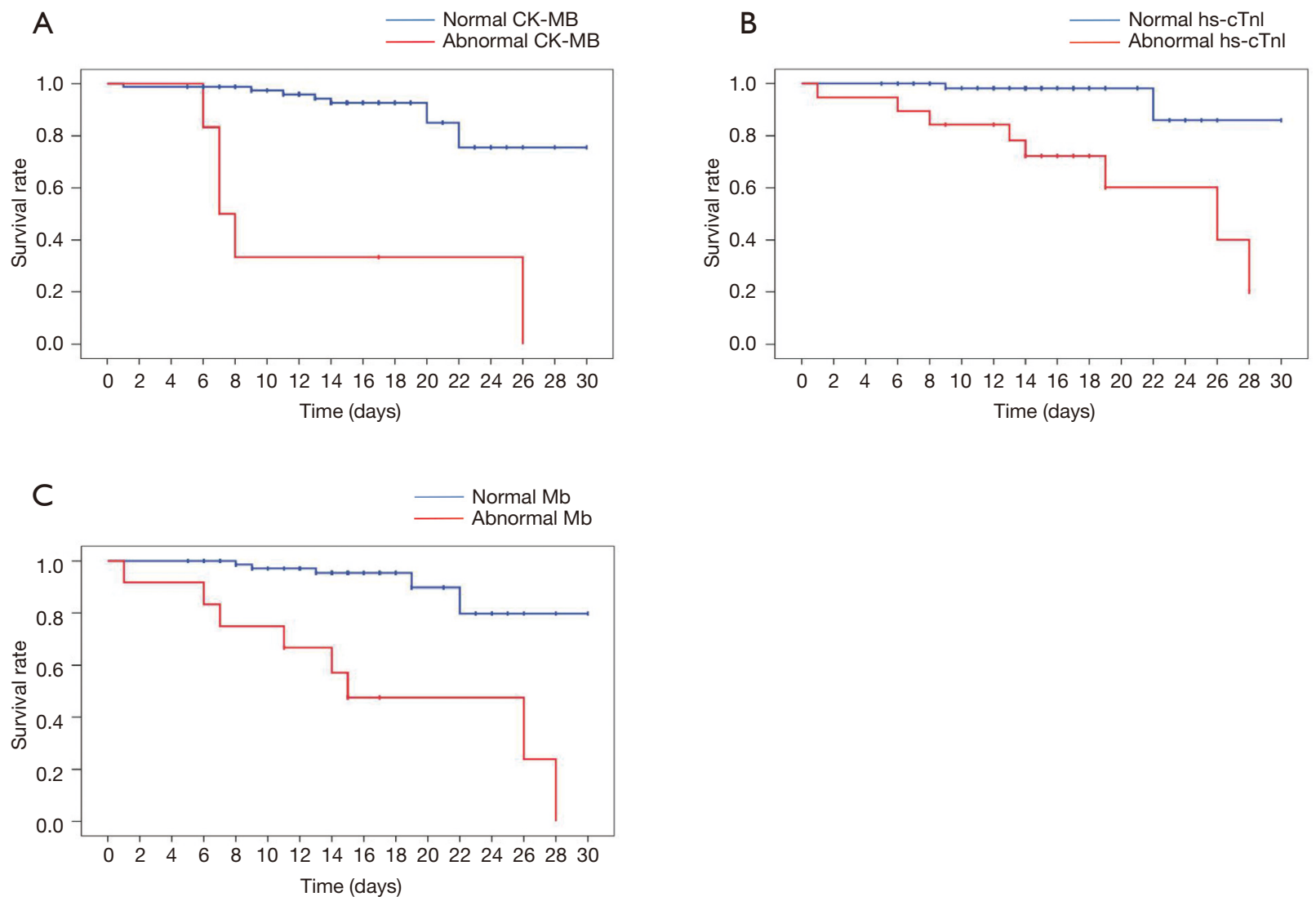

Figure 1 Kaplan-Meier survival analysis for critically ill COVID-19 patients with different levels of CK-MB (A), hs-cTnI (B) and Mb (C). COVID-19, coronavirus disease 2019; CK-MB, creatine kinase isoenzyme-MB; hs-cTnI, high-sensitivity cardiac troponini I; Mb, myoglobin.

with older age and chronic comorbidity tend to have poor prognosis $(18,19)$. Inconsistent with this, we also noticed that the non-survivors average age was markedly higher than the survivors. Simultaneously, we did not find statistical differences in the incidence of hypertension, diabetes, coronary heart disease, and chronic obstructive pulmonary disease between non-survivors and survivors. This paradox might be attributed to patient homogeneity (all the patients were critically ill cases); thus, it is reasonable to believe that the critically ill COVID-19 patients had more chronic comorbidities than the mild COVID-19 patients.

Compared with the survivors, we also noticed elevated levels of AST, total bilirubin, urea nitrogen, serum creatinine, hsCRP, and myocardium injury biomarkers in the non-survivors. These findings reflected the severity of COVID-19 and were inconsistent with the notion that COVID-19 caused multiple organ injury. Nevertheless, we found they do not have equal efficacy in predicting the prognosis. According to the multivariate Cox regression analysis, only age, lymphocyte count, and total bilirubin were associated with the prognosis. In agreement with this, these indexes were included in Apache- II and SOFA scoring system to evaluate disease severity in critically ill patients.

During our clinical practice in Wuhan, we also noticed an increase in myocardium injury biomarkers in the early phase of COVID-19. Patients who developed COVID19-associated myocardium injury showed an inferior prognosis. This might be attributed to the expression of ACE2 in the myocardium cells, which leads to direct attack from the virus and secondary attack from the activated immune cells, respectively (20). Interestingly, most patients were asymptomatic, whereas blood biochemical test may reveal an increase in myocardium injury biomarkers. In 


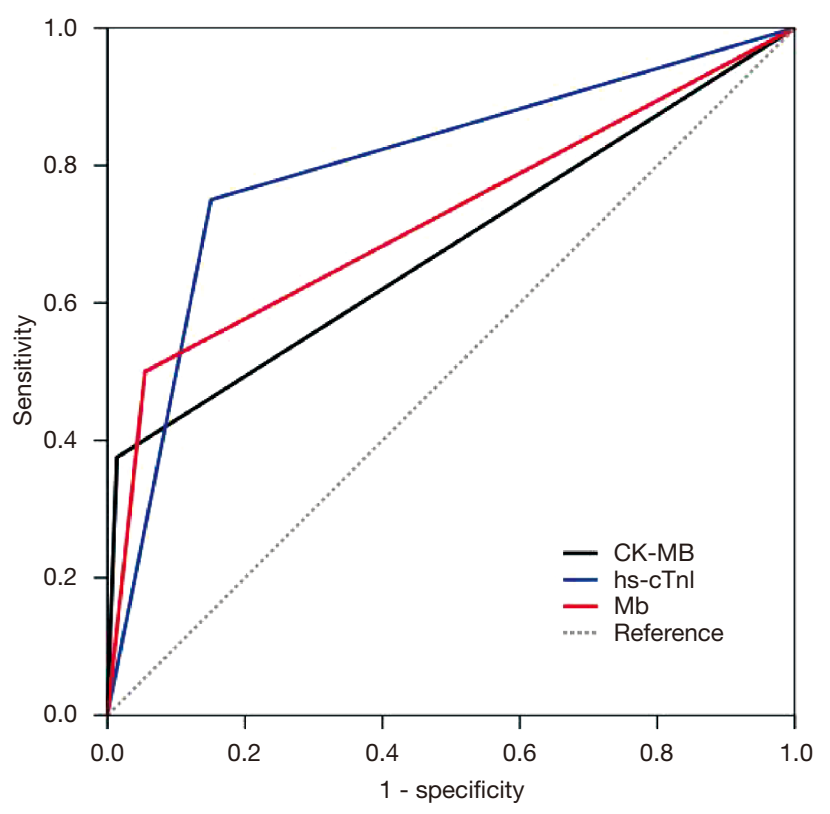

Figure 2 Evaluation of the sensitivity and the specificity of myocardium injury biomarkers in predicting the prognosis of critically ill COVID-19 patients. COVID-19, coronavirus disease 2019; CK-MB, creatine kinase isoenzyme-MB; hs-cTnI, highsensitivity cardiac troponini I; Mb, myoglobin.

this retrospective study, we found that $54.5 \%(12 / 22)$ patients had an elevated level of CK-MB in the nonsurvivors, whereas only 1 (1.3\%) patient developed CKMB abnormality in the survivors. Of the 78 who survived critically ill COVID-19 patients, 11 (14.1\%) patients, and $3(3.8 \%)$ patients developed abnormalities in hs-c TnI and Mb. However, this occurred in 17 (77.2\%) patients and $13(59.1 \%)$ patients in the non-survivors. Echocardiography examination may also indicate ventricular premature beats and decrease in ejection fraction (unpublished data). To this end, it is not surprising to notice that critically ill COVID-19 patients with elevated level myocardium injury biomarkers tended to have unfavorable survival.

Next, we determined whether these myocardium injury biomarkers serve as prognostic factors for critically ill COVID-19 patients, while we found they do not have equally prognostic significance. Although univariate analysis yields $\mathrm{P}$ values less than 0.05 for CK-MB, hs-cTnI, and $\mathrm{Mb}$, two multivariate analysis models converge at hs-c TnI (Table 3). More importantly, hs-cTnI possess the highest sensitivity in predicting prognosis. The abnormality in the hs-cTnI level indicates myocardium injury and is associated with the disease's severity. Since the COVID-19 pandemic is still ongoing, early recognition and timely intervention would reduce critically ill COVID-19 patients' mortality.

Since there is no specific treatment for COVID-19 at the moment, management of COVID-19-associated myocardium injury is largely supportive. According to our clinical experience in Wuhan, $\beta$-receptor blockers may be used to slow down the heart rate, and diuretic drugs could reduce liquid burden. Several myocardial nutraceuticals, including trimetazidine and coenzyme Q10, were also used in the clinical practice. We speculated that more potent and specific strategy for COVID-19, such as convalescent plasma and SARS-CoV-2 vaccines, would be much more efficient.

This study had several limitations. Firstly, the retrospective and single-center nature might lead to some bias, which might be inevitable; however, the quality control was ensured because the same experienced team completed all administrations. Secondly, the sample size might be limited; yet we adjusted the effect of myocardium injury indicators in two separate multivariate models to ensure the findings' accuracy and stability. Finally, all patients in our study were Chinese with the critically ill disease; thus, extrapolation and generalization of our findings should be cautious, and future studies are needed.

In conclusion, the age, lymphocyte count, total bilirubin concentration, and myocardium injury biomarkers are independent prognostic factors for critically ill COVID-19 patients, among which hs-cTnI might be a novel and sensitive indicator. This indicator should be given special attention, and time-intensive treatment should be considered in such patients.

\section{Acknowledgments}

We greatly appreciate all front-line health care colleagues in Wuhan. We express gratitude to all the participants and their families.

Funding: This study was sponsored by the Key Project of Social Development, Shaanxi Province (\#2017ZDXMSF-049 and \#2020JQ-863), the National Natural Science Foundation of China (\#81803950), the Subject Innovation Team of Shaanxi University of Chinese Medicine (\#2019YS01), and the Xi'an Fourth Hospital Research Incubation Fund (\#FZ-35).

\section{Footnote}

Reporting Checklist: The authors have completed the STARD 
reporting checklist. Available at http://dx.doi.org/10.21037/ apm-20-2112

Data Sharing Statement: Available at http://dx.doi. org/10.21037/apm-20-2112

Conflicts of Interest: All authors have completed the ICMJE uniform disclosure form (available at http://dx.doi. org/10.21037/apm-20-2112). The authors have no conflicts of interest to declare.

Ethical Statement: The authors are accountable for all aspects of the work in ensuring that questions related to the accuracy or integrity of any part of the work are appropriately investigated and resolved. The study was conducted in accordance with the Declaration of Helsinki (as revised in 2013). The Ethics Medical Committee approved this study of Hubei Women \& Children Healthcare Hospital (No.2020-FYGG-011). Written consent was obtained from each participant.

Open Access Statement: This is an Open Access article distributed in accordance with the Creative Commons Attribution-NonCommercial-NoDerivs 4.0 International License (CC BY-NC-ND 4.0), which permits the noncommercial replication and distribution of the article with the strict proviso that no changes or edits are made and the original work is properly cited (including links to both the formal publication through the relevant DOI and the license). See: https://creativecommons.org/licenses/by-nc-nd/4.0/.

\section{References}

1. Roussel Y, Giraud-Gatineau A, Jimeno MT,et al. SARSCoV-2: fear versus data. Int J Antimicrob Agents 2020;55:105947.

2. Ou X, Liu Y, Lei X, et al. Characterization of spike glycoprotein of SARS-CoV-2 on virus entry and its immune cross-reactivity with SARS-CoV. Nat Commun 2020;11:1620.

3. Liu Z, Xiao X, Wei X, et al. Composition and divergence of coronavirus spike proteins and host ACE2 receptors predict potential intermediate hosts of SARS-CoV-2. J Med Virol 2020;92:595-601.

4. Zheng M, Song L. Novel antibody epitopes dominate the antigenicity of spike glycoprotein in SARSCoV-2 compared to SARS-CoV. Cell Mol Immunol
2020;17:536-8.

5. Ye M, Fu D, Ren Y, et al. Treatment with convalescent plasma for COVID-19 patients in Wuhan, China. J Med Virol 2020. [Epub ahead of print].

6. Gao Y, Yan L, Huang Y, et al. Structure of the RNAdependent RNA polymerase from COVID-19 virus. Science 2020;368:779-82.

7. Liu J, Zheng $X$, Tong $\mathrm{Q}$, et al. Overlapping and discrete aspects of the pathology and pathogenesis of the emerging human pathogenic coronaviruses SARS-CoV, MERS-CoV, and 2019-nCoV. J Med Virol 2020;92:491-4.

8. Letko M, Marzi A, Munster V. Functional Assessment of Cell Entry and Receptor Usage for SARS-CoV-2 and Other Lineage B Betacoronaviruses. Nat Microbiol 2020;5:562-9.

9. Feng G, Zheng K, Yan QQ, et al. COVID-19 and Liver Dysfunction: Current Insights and Emergent Therapeutic Strategies. J Clin Transl Hepatol 2020;8:18-24.

10. Rovin BH, Ronco P. Kidney International and the COVID-19 Infection. Kidney Int 2020;97:823.

11. Akhmerov A, Marbán E. COVID-19 and the Heart. Circ Res 2020;126:1443-55.

12. Li YC, Bai WZ, Hashikawa T. The neuroinvasive potential of SARS-CoV2 may play a role in the respiratory failure of COVID-19 patients. J Med Virol 2020;92:552-5.

13. Hanley B, Lucas SB, Youd E, et al. Autopsy in suspected COVID-19 cases. J Clin Pathol 2020;73:239-42.

14. Mo $P$, Xing $Y$, Xiao Y, et al. Clinical characteristics of refractory COVID-19 pneumonia in Wuhan, China. Clin Infect Dis 2020. [Epub ahead of print].

15. Wang K, Zuo P, Liu Y, et al. Clinical and Laboratory Predictors of In-Hospital Mortality in Patients With COVID-19: A Cohort Study in Wuhan, China. Clin Infect Dis 2020;71:2079-88.

16. Shi S, Qin M, Shen B, et al. Association of Cardiac Injury With Mortality in Hospitalized Patients With COVID-19 in Wuhan, China. JAMA Cardiol 2020;5:802-10.

17. Grasselli G, Zangrillo A, Zanella A, et al. Baseline Characteristics and Outcomes of 1591 Patients Infected With SARS-CoV-2 Admitted to ICUs of the Lombardy Region, Italy. JAMA 2020;323:1574-81.

18. Wang M, Guo L, Chen Q, et al. Typical radiological progression and clinical features of patients with coronavirus disease 2019. Aging (Albany NY) 2020;12:7652-9.

19. Chen N, Zhou M, Dong X, et al. Epidemiological and Clinical Characteristics of 99 Cases of 2019 Novel 
Coronavirus Pneumonia in Wuhan, China: A Descriptive Study. Lancet 2020;395:507-13.

20. Yang J, Li H, Hu S, et al. ACE2 Correlated With Immune Infiltration Serves as a Prognostic Biomarker in Endometrial Carcinoma and Renal Papillary Cell

Cite this article as: Cao L, Zhang S, Luo X, Wang E, Bai Y, Li Z, Li F, Ma J, Liu H. Myocardium injury biomarkers predict prognosis of critically ill coronavirus disease 2019 (COVID-19) patients. Ann Palliat Med 2020;9(6):4156-4165. doi: 10.21037/ apm-20-2112
Carcinoma: Implication for COVID-19. Aging (Albany NY) 2020;12:6518-35.

(English language editor: J. Chapnick) 